\title{
Breakup of dipolar rings under a perpendicular magnetic field
}

\author{
F. Kun, ${ }^{1,2, *}$ Weijia Wen, ${ }^{3}$ K. F. Pál, ${ }^{4}$ and K. N. Tu ${ }^{5}$ \\ ${ }^{1}$ Department of Theoretical Physics, University of Debrecen, P. O. Box 5, H-4010 Debrecen, Hungary \\ ${ }^{2}$ Institute for Computer Applications (ICA1), University of Stuttgart, D-70569 Stuttgart, Germany \\ ${ }^{3}$ Department of Physics, The Hong Kong University of Science and Technology, Clear Water Bay, Kowloon, Hong Kong \\ ${ }^{4}$ Institute of Nuclear Research (ATOMKI), P. O. Box 51, H-4001 Debrecen, Hungary \\ ${ }^{5}$ Department of Materials Sciences and Engineering, University of California at Los Angeles, Los Angeles, California $90095-1595$
}

(Received 5 July 2001; published 20 November 2001)

\begin{abstract}
An experimental and theoretical study of the breakup process of rings, formed by magnetic microspheres, under the application of an external magnetic field perpendicular to the plane of the ring is presented. We found experimentally that when the value of the external magnetic field falls below a lower critical field the dipoles rotate in the ring without any distortion of the ring structure. However, exceeding the upper critical field causes sudden breakup of the ring into short chains aligned with the field. Between the lower and upper critical fields the system is in a metastable state, and hence, it is very sensitive to external perturbations. The spiral opening was found experimentally to be the lowest energy transition from the ring to the chain conformation. We worked out an analytic approach and we performed computer simulations, the results of which are in good agreement with experiments.
\end{abstract}

DOI: 10.1103/PhysRevE.64.061503

PACS number(s): 83.10.Pp, 82.70.Dd, 41.20.-q, 61.46.+w

\section{INTRODUCTION}

In colloidal materials composed of micrometer-sized magnetic particles suspended in a nonmagnetic viscous liquid, in the absence of an external magnetic field the magnetic particles aggregate due to the interplay of the dipole-dipole interaction and of the Brownian motion of the particles, and they build up complex structures. The intriguing effect of long-range dipolar forces on the dynamics of growth processes and on the structure of growing aggregates in colloids have attracted much scientific and industrial interest during the past years [1-12].

The formation of structures of magnetic particles and their disintegration under various kinds of external perturbations is a very interesting issue that has initiated intensive research. In these investigations the study of the behavior of small regular structures, like chains, or rings composed of a few particles, is essential since they can serve as a starting point for the understanding of more complicated conformations. Due to this reason the formation of chains and columns of magnetic particles in an external magnetic field has been extensively studied [1-7]. Very recently, the micromechanical properties, the deformation and rupturing of dipolar chains and columns, was investigated [8-11]. Another important structure of dipolar particles is the circular ring, which provides the lowest energy conformation when the number of particles is larger than 4 . Recently, we reported an experimental and theoretical investigation of the formation of two-dimensional circularly shaped rings of dipoles in colloidal materials in the absence of an external magnetic field, and that of the competition of rings with randomly oriented open chains and labyrinthine structures when changing the volume fraction of particles [12]. We have analyzed the stability of rings of dipoles with respect to external mechanical

*Electronic address: feri@dtp.atomki.hu perturbations by analytic means, and we also tested experimentally the stability of structures formed in the absence of an external field against vibrations [12]. The deformation and breakup process of dipolar rings under the application of an external magnetic field parallel to the ring's plane was studied in Ref. [13].

Subjecting a dipolar ring, formed in a suspension, to a weak external magnetic field $B$, not sufficient to destroy the structure, the ring rotates until its plane gets perpendicular to the field achieving equilibrium. However, a further increase of the field value can cause the breakup of the ring structure. The problem of the breakup of a planar ring of dipoles under the application of an external magnetic field perpendicular to the plane of the ring has been addressed in Ref. [14], where a simplified analytic approach has been worked out, combined with computer simulations, but no experimental results have been presented. The analytic approach of Ref. [14] allowed for solely nearest neighbor interactions between the particles of the ring. To capture the effect of the long-range forces a parameter had to be introduced, the value of which was determined by fitting the analytic formulas to the results of computer simulations.

In this paper we reconsider this problem and we present for the first time a thorough experimental and theoretical investigation of the breakup process of dipolar rings subjected to an external magnetic field perpendicular to the plane of the ring. We found experimentally that when the value of the external magnetic field $B$ falls below a lower critical field $B_{\mathrm{c}}^{1}$ the dipoles rotate in the ring without any distortion of the ring structure. However, exceeding an upper critical field $B_{\mathrm{c}}^{\mathrm{u}}$ causes the sudden breakup of the ring into short chains aligned with the field. Between the lower and upper critical fields the system is in a metastable state, and hence it is very sensitive to external perturbations. The spiral opening was found experimentally to be the lowest energy transition from the ring to the chain conformation. We worked out an analytic approach treating long-range interac- 


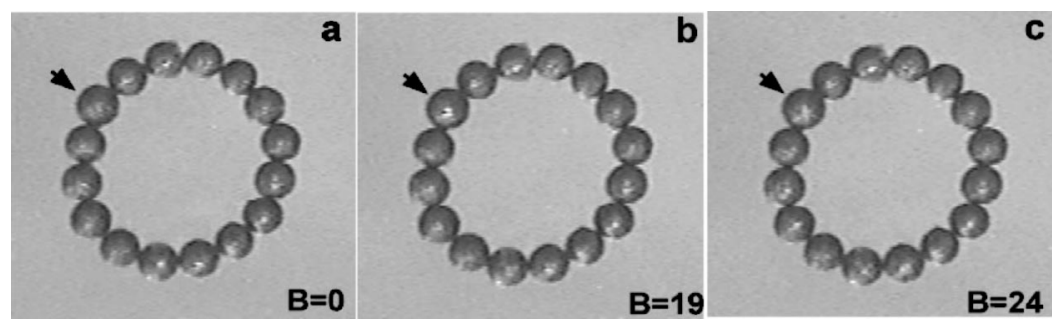

FIG. 1. Top view of a ring of dipoles. One can observe the rotation of the marked particle.

tions correctly, and we could relate the characteristic quantities of the break-up process with that of the initial ring. Experiments, analytic theory, and computer simulations are in satisfactory agreement. The role of friction between the particles and between the particles and the bottom plate of the vessel are discussed; furthermore, the effect of gravity and that of the lateral constraint due to the hard ground of the vessel are discussed. The effect of polarization of the particles due to the external magnetic field and the mutual dipolar fields is analyzed.

\section{EXPERIMENTS}

For the experimental study of the breakup process the circularly shaped rings of dipolar particles have been produced as described in Refs. [12,13]. The momentcontrollable magnetic particles were fabricated by selecting uniform glass microspheres with an average diameter of 47 $\mu \mathrm{m}$ as an initial core, and by coating a layer of nickel of thickness about $3.3 \mu \mathrm{m}$ using a chemical coating process [15]. The magnetization $M$ of microspheres used in our experiments is $480 \mathrm{emu} / \mathrm{cm}^{3}$. In the experiments the optical side of a 2-in. Si wafer was used as the bottom plate on which four plastic barriers are mounted to form a container filled with silicone oil of viscosity $\eta=517.68 \mathrm{mPas}$. The container was placed in the center region of a pair of Helmholz coils, where the magnetic strength of coils was controlled by a current amplifier. The pattern evolution of microspheres in the container was monitored in situ by a charge-coupled device (CCD) camera and a video recorder. First, the nickel-coated microspheres were magnetized and then dispersed randomly onto the container in the absence of an external magnetic field. The particles settle down onto the bottom plate of the vessel and organize themselves into various planar structures; they also form planar rings. In Ref. [12] we pointed out that the formation of circularly shaped rings on the bottom plate of the vessel is due to the relatively large particles size, which hinders the Brownian motion of the particles at room temperature and provides a magnetic coupling the strength of which is much larger than the thermal energy.

After the formation of the circularly shaped rings of magnetic microspheres, those rings were selected for further studies which occurred far from the other structures in the colloid in order to minimize the disturbing effect of the surroundings. In the next stage of the experiments, the external magnetic field was switched on such that its direction was fixed to be perpendicular to the plane of the rings. The magnitude of $B$ was controlled in two different ways: $B$ was increased linearly with time starting from 0 and, further- more, experiments have been performed by suddenly switching on the magnetic field and keeping its value fixed.

When the magnitude of $B$ was increased slowly, we have observed that the particles rotated in the ring without distortion of the ring's structure. The rotation of a particle is demonstrated in Fig. 1, where the rotational status of the microsphere pointed by the arrow can be clearly seen due to the changes of the bright spark on it. In Fig. 1(a) there is no spark on the microsphere, while increasing the magnetic field intensity the bright spark appears when the ball rotated, which can be seen in Figs. 1(b) and 1(c). However, the rotation of particles appeared at random positions along the ring and they were found to be discontinuous, i.e., rotations occurred in sudden jumps under the slowly increasing field. This effect is caused by the sticking friction between the particles and between the particles and the bottom plate of the vessel.

When $B$ exceeds a threshold value under slow increase, the ring opens up at a random position, i.e., a particle starts to move in the direction of the field and the whole ring opens in a spiral way as presented in Fig. 2. If $B$ was kept fixed the opening process stopped due to the gravitational force acting on the particles, and the particle system stabilized itself in the actual conformation. To get the whole chain of particles aligned with the field, the magnitude of $B$ had to be continuously increased. Under slowly increasing magnetic field only the spiral opening was found experimentally as a transition mechanism from the ring to the chain conformation. In Fig. 2 the opening started at about 20-25 G and the field had to be increased up to $40 \mathrm{G}$ to get full alignment.

When a magnetic field $B$ of constant value was imposed suddenly, the specific field value was searched for, which causes the immediate breakup of the ring. Typical results of these experiments are presented in Fig. 3 where the time evolution of a ring can be seen after switching on the magnetic field. In this case the magnetic field $B$ is strong enough to fix the dipole moments parallel to each other which gives rise to an explosion of the ring. Afterwards, the separated particles gather again and form short chains along the field due to the dipole-dipole interaction. The minimum value of $B$ needed to provoke immediate breakup of the ring was found to be between 40 and $50 \mathrm{G}$ for rings of 10-20 particles.

\section{ANALYTIC APPROACH}

The problem of the breakup of rings under an external magnetic field perpendicular to the plane of the ring can be studied analytically up to some extent. A simplified analytic treatment has been performed in Ref. [14], where only nearest neighbor interactions have been considered between the 

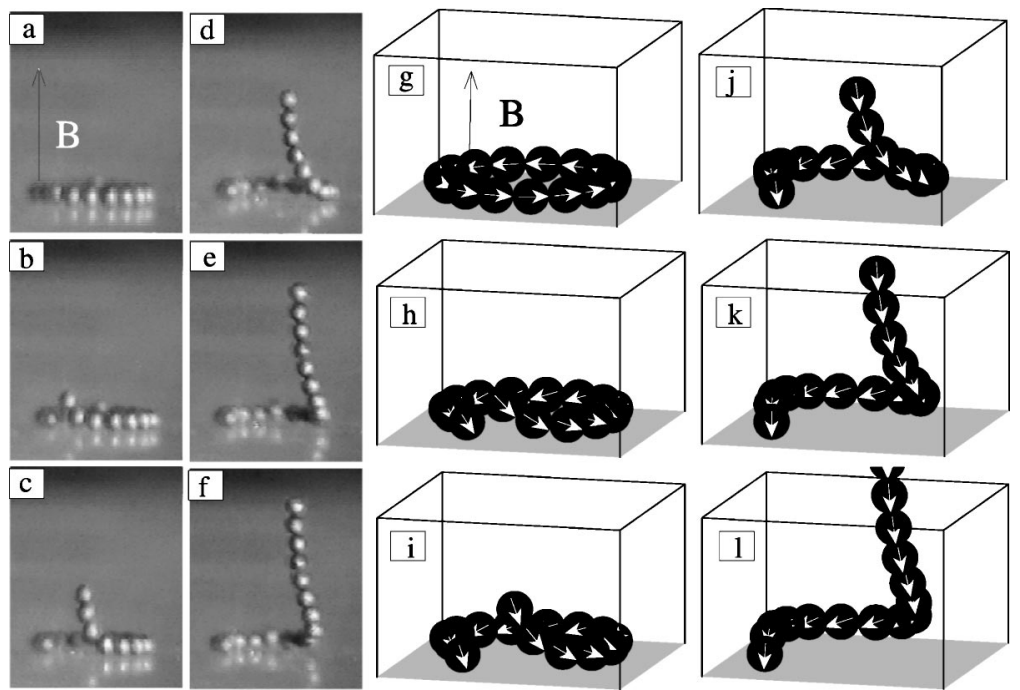

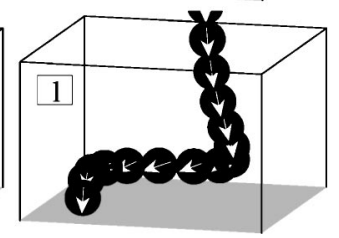

FIG. 2. In the experiments, the ring opens in a spiral way into a chain and aligns with the external fields (a)-(f). The magnitude of $B$ had to be increased during the opening process to overcome the gravitational force acting on the particles. Computer simulations are in good agreement with the experiments $(\mathrm{g})-(1)$. In snapshots of the simulations the arrows indicate the orientation of the dipole moments. particles. To capture the long-range nature of the dipolar interaction a parameter was introduced into the analytic model, the value of which was determined by fitting the analytic results to the exact numerical solution obtained by computer simulations. In the following we do not restrict the dipoledipole interaction to nearest neighbors but we treat the longrange interaction correctly. This way the analytic results are obtained in closed form without any fitting parameter; furthermore, this formulation allows us to directly relate the characteristic quantities of the breakup process to those of the initial ring.

In the theoretical treatment the system under consideration is modeled as a monodispersive ensemble of soft particles of number $N$ and magnetic moment $\mu$. The particles are represented by spheres of radius $R$ (diameter $d$ ) and pointlike dipole moments are assigned to their center, which corresponds to the assumption of a homogeneous distribution of dipole moments in a spherical shell of uniform thickness neglecting any spatial inhomogeneities.

It can be simply shown that in the absence of an external magnetic field the lowest energy configuration of dipoles, if the number of dipoles is larger than 4 , is the ring structure which is illustrated in Fig. 4 [12]. Compared to a straight chain of aligned dipoles in the absence of an external magnetic field the ring is energetically more favorable because the closing of the two ends of the chain overweights the energy loss due to the misalignment of dipoles. In the ring configuration of $N$ dipoles, all the dipole moments lie in the ring's plane and the angle $\beta$, enclosed by the direction of a dipole and the line connecting the center of two consecutive particles in the ring, can be given as $\beta=\pi / N$ [12].

If a weak external magnetic field is switched on perpendicular to the plane of the ring, the dipole moments of the particles turn out of the ring's plane in the direction of the external field but the ring structure remains unchanged. Due to the symmetry of the system, all the dipoles have the same rotation and the dipole moments are always tangential to the surface of a cylinder defined by the initial ring and the external magnetic field. Let us denote by $\Theta$ the angle between the orientation of dipoles and the external magnetic field $B$, i.e., $0 \leqslant \Theta \leqslant \pi / 2$. The potential energy of the ring of dipoles divided by the number of particles $N$ can be obtained as

$$
W=\frac{1}{N} \sum_{i>j} u_{i j}-\mu B \cos \Theta .
$$

In Eq. (1) $u_{i j}$ is the interaction energy of two dipoles $\mu \vec{e}_{i}$ and $\mu \vec{e}_{j}$ separated by the distance $r_{i j}$

$$
u_{i j}=\frac{\mu^{2}}{r_{i j}^{3}}\left[\vec{e}_{i} \cdot \vec{e}_{j}-3\left(\vec{e}_{i} \cdot \vec{n}_{i j}\right)\left(\vec{e}_{j} \cdot \vec{n}_{i j}\right)\right]
$$

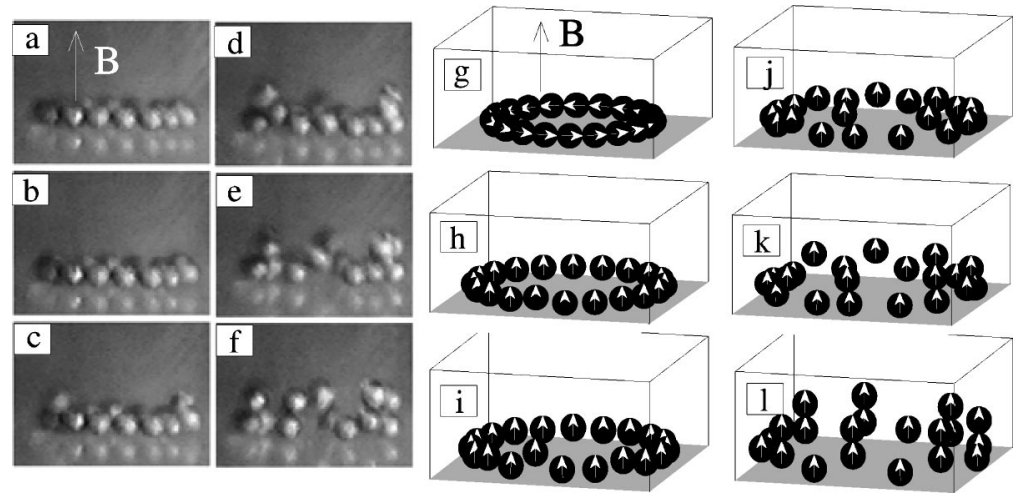

FIG. 3. The breakup of a ring under a constant magnetic field $B$ imposed suddenly. The experimental results (a)-(f) are in good agreement with the simulations $(\mathrm{g})-(\mathrm{l})$. 


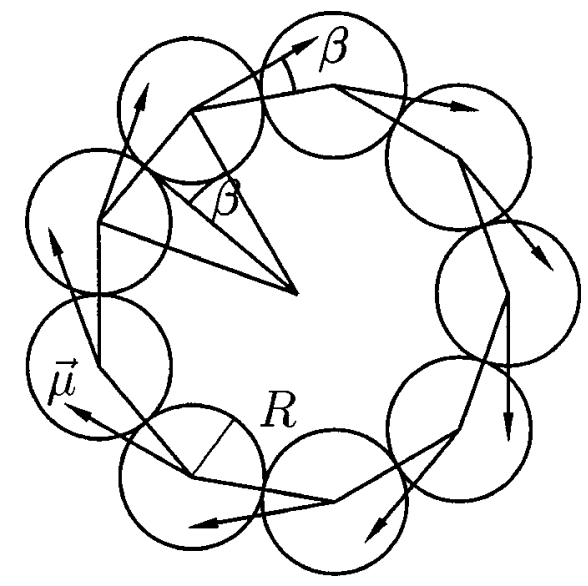

FIG. 4. The ring of dipoles when pointlike dipoles $\vec{\mu}$ are assigned to the center of spherical particles of radius $R$. $\beta$ denotes the equilibrium angle between the dipole moments and the connecting line of consecutive particles in the ring.

where $\vec{n}_{i j}$ denotes the unit vector pointing from dipole $i$ to dipole $j$. Until the ring structure is undistorted, the orientation of the dipole moment of particle $i$ can be represented in spherical coordinates as $\vec{e}_{i}$ $=\left(\sin \Theta \cos \phi_{i}, \sin \Theta \sin \phi_{i}, \cos \Theta\right)$, where $\phi_{i}=2 i \beta$ characterizes the position of the dipole along the ring. The distance $r_{i j}$ of particles $i$ and $j$ can be obtained as $r_{i j}=d \sin [(i$ $-j) \beta] / \sin \beta$. Based on these formulas the summation in Eq. (1) can be rewritten, and the energy of the ring $W$ can be cast in the following form:

$$
\begin{aligned}
W= & -\frac{\mu^{2} \sin ^{3} \beta}{N d^{3}} \sum_{i=1}^{N-1}(N-i) \frac{1+\cos ^{2}(i \beta)}{\sin ^{3}(i \beta)} \\
& +\cos ^{2} \Theta \frac{\mu^{2} \sin ^{3} \beta}{N d^{3}} \sum_{i=1}^{N-1}(N-i) \frac{2+\cos ^{2}(i \beta)}{\sin ^{3}(i \beta)} \\
& -\mu B \cos \Theta .
\end{aligned}
$$

It can be recognized that the first term in Eq. (3) is the energy $W_{0}^{\mathrm{r}}$ of a ring of $N$ particles in the absence of an external magnetic field, see also Ref. [12]. The second term accounts for the increase of the dipole-dipole interaction energy due to the rotation of dipoles out of the ring's plane, and the third term provides the energy due to the interaction with the external field. Hence, Eq. (3) can be simplified to

$$
W=W_{0}^{\mathrm{r}}+K \cos ^{2} \Theta-\mu B \cos \Theta
$$

where $K>0$ is solely determined by the initial ring structure according to Eq. (3). The equilibrium angle $\Theta_{\mathrm{e}}$ can be obtained by minimizing $W$ with respect to $\Theta$ at a fixed magnetic field $B$

$$
\cos \Theta_{\mathrm{e}}=\frac{\mu B}{2 K},
$$

and the energy takes the final form

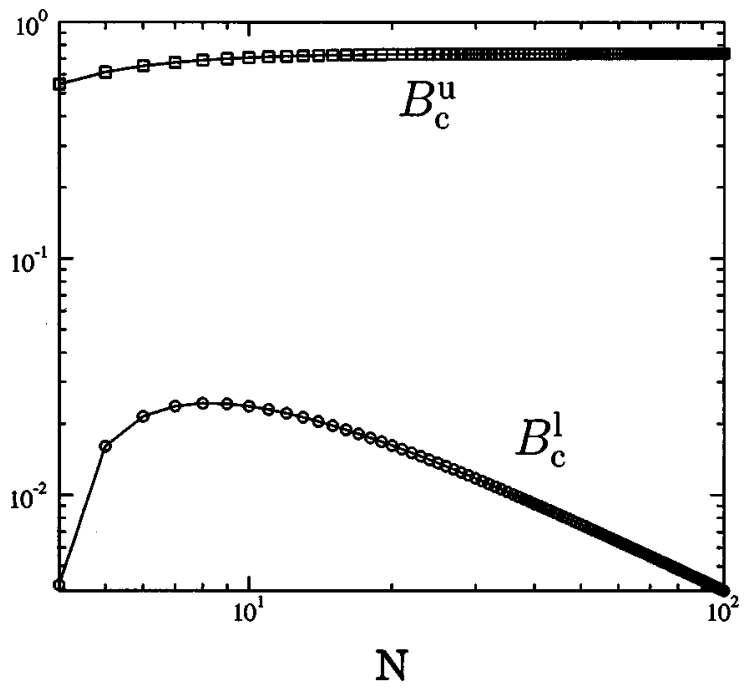

FIG. 5. The upper $B_{\mathrm{c}}^{\mathrm{u}}$ and lower $B_{\mathrm{c}}^{1}$ critical fields as a function of the number of particles $N$ of the ring in dimensionless form.

$$
W=W_{0}^{\mathrm{r}}-\frac{\mu^{2} B^{2}}{4 K}
$$

Increasing the external field $B$, the ring configuration loses its stability at a critical field $B_{\mathrm{c}}^{\mathrm{u}}$ corresponding to a specific value of the rotation angle $\Theta_{c}$ at which the energy of the dipole-dipole interaction, i.e., the sum of the first two terms in Eq. (4), becomes positive. From Eqs. (4)-(6) it follows

$$
B_{\mathrm{c}}^{\mathrm{u}}=\frac{2}{\mu} \sqrt{-W_{0}^{\mathrm{r}} K}, \quad \cos \Theta_{\mathrm{c}}=\sqrt{-\frac{W_{0}^{\mathrm{r}}}{K}} .
$$

$B_{\mathrm{c}}^{\mathrm{u}}$ is called the upper critical field, see also Ref. [14]. Note that the above analytic expressions of the energy hold until the ring structure persists, i.e., up to $B_{\mathrm{c}}^{\mathrm{u}}$.

For the particle system the chain configuration aligned with the field becomes energetically favorable already at a lower value of the external field $B_{\mathrm{c}}^{1}$, which is called the lower critical field. Since the energy of a chain aligned with $\vec{B}$ is $W^{\mathrm{c}}=W_{0}^{\mathrm{c}}-\mu B$, the lower critical field reads as

$$
B_{\mathrm{c}}^{1}=\frac{2 K}{\mu}\left[1-\sqrt{1+\frac{1}{K}\left[W_{0}^{\mathrm{r}}-W_{0}^{\mathrm{c}}\right]}\right],
$$

where $W_{0}^{\mathrm{c}}$ denotes the energy of a chain in the absence of an external field. The upper and lower critical fields are illustrated in Fig. 5 as a function of $N$, where the unit of $B$ is $B_{0}=\mu / R^{3}$. It can be observed that $B_{\mathrm{c}}^{\mathrm{u}}$ saturates for large $N$, while $B_{\mathrm{c}}^{1}$ has a maximum at $N=8$ and it goes to zero for large $N$, since in this limit the difference between the ring and chain configurations disappears. The critical angle $\Theta_{c}$ as a function of $N$ is presented in Fig. 6. The saturation value of $\Theta_{\text {c }}$ for large $N$ corresponds to the critical angle of an infinite chain.

It can be considered that when the external field $B$ falls between $B_{\mathrm{c}}^{1}$ and $B_{\mathrm{c}}^{\mathrm{u}}$ the ring is in a metastable state, which also implies that it is sensitive to external perturbations. The 


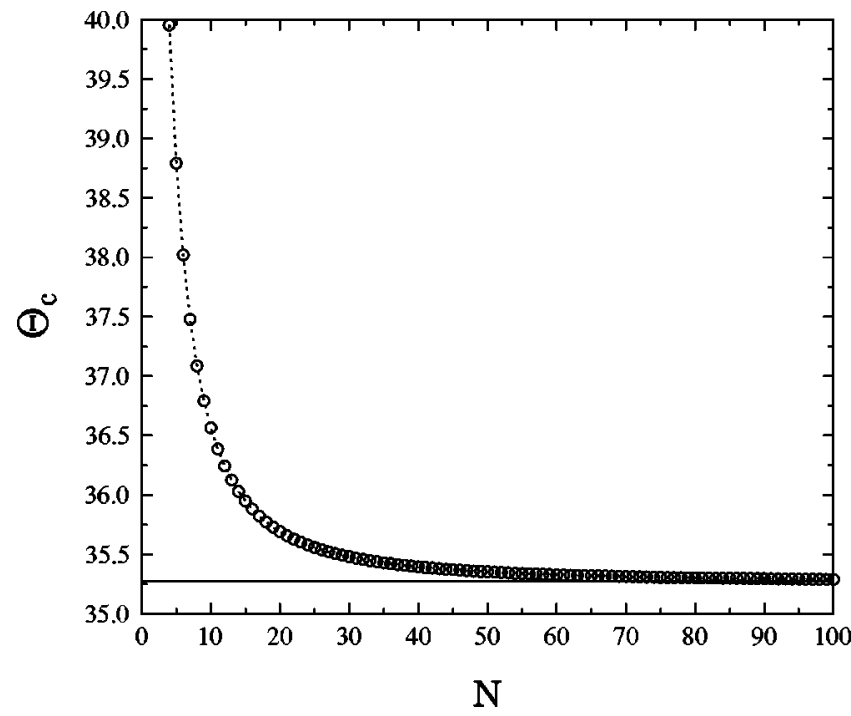

FIG. 6. The critical angle in degrees at which sudden breakup occurs. The horizontal continuous line indicates the limit angle, where the interaction of two dipoles becomes repulsive when the two dipole vectors and the line connecting the two particles lie in the same plane, i.e., in a chain configuration.

response of the system to external perturbations for $B_{\mathrm{c}}^{1}<B$ $<B_{\mathrm{c}}^{\mathrm{u}}$ can only be explored by means of computer simulations.

\section{COMPUTER SIMULATIONS}

A deeper insight into the breakup process can be obtained by means of computer simulations. In the simulations the model used in the analytic part is completed to make possible the study of the time evolution of the breakup process. The two-dimensional version of the model has been successfully used in our recent works $[12,13]$. In general, the spherical particles in the model have six continuous degrees of freedom in three dimensions, i.e., the three coordinates of the center of mass and the three Euler angles characterizing the orientation of the dipole moment.

The time evolution of the particle system is followed by solving the equations of motion for the translational degrees of freedom of the particles (molecular dynamics [16]), and by applying a self-consistent relaxation technique to capture the rotational motion. The particles are subjected to the dipole-dipole and dipole-external field interaction, to hydrodynamic resistance due to their motion relative to the liquid phase, and to an elastic restoring force (soft particle dynamics) in order to take into account the finite size of the particles and the interaction with the hard bottom plate of the container in which the pattern evolution is studied. Motivated by the experimental observations, no stochastic forces are taken into account (no thermal motion) in the simulations.

The magnetic force $\vec{F}_{i j}^{m}$ acting between two dipoles $\mu \vec{e}_{i}$ and $\mu \vec{e}_{j}$ separated by a distance $r_{i j}$ is supposed to have the form $\vec{F}_{i j}^{m}=\mu^{2} \vec{f}_{i j}^{m}$ with

$$
\begin{aligned}
\vec{f}_{i j}^{m}= & \frac{3}{r_{i j}^{4}}\left[5\left(\cos \beta_{i}\right)\left(\cos \beta_{j}\right) \vec{n}_{i j}-\cos \left(\beta_{i}-\beta_{j}\right) \vec{n}_{i j}\right. \\
& \left.-\vec{e}_{i} \cos \beta_{j}-\vec{e}_{j} \cos \beta_{i}\right],
\end{aligned}
$$

where $\vec{n}_{i j}$ denotes the unit vector pointing from dipole $i$ to dipole $j$, and $\beta_{i}, \beta_{j}$ are the angles of the direction of the dipoles with respect to $\vec{n}_{i j}$. The hydrodynamic force $\vec{F}_{i}^{\text {hyd }}$ on a sphere is treated as Stockes's drag $\vec{F}_{i}^{\text {hyd }}=-\alpha \vec{v}_{i}$, where $\vec{v}_{i}$ denotes the velocity of particle $i$ and $\alpha=6 R \pi \eta$. The particles are assumed to be deformable bodies which can overlap each other during their motion representing local deformations up to some extent. To capture the finite size of the particles, an elastic restoring force is introduced between overlapping particles according to Hertz's contact law [17]

$$
\vec{F}_{i j}^{\mathrm{pp}}=-k_{\mathrm{pp}}\left(d-r_{i j}\right)^{3 / 2} \vec{n}_{i j}=-k_{\mathrm{pp}} \vec{f}_{i j}^{\mathrm{pp}}
$$

where $k$ is a material-dependent constant, and the superscript "pp" refers to the particle-particle contact. A similar contact force is introduced between the particles and the bottom plate of the vessel $\vec{F}_{i}^{\mathrm{pg}}=-k_{\mathrm{pg}} \vec{f}_{i}^{\mathrm{pg}}$ in order to ensure the constraint by the hard ground. For simplicity, no gravitational force was implemented on the particles.

The system is supposed to be fully dissipative and noninertial, hence

$$
\begin{gathered}
\frac{d \vec{r}_{i}}{d t}=\frac{\mu^{2}}{\alpha} \sum_{j} \vec{f}_{i j}^{\mathrm{m}}-\frac{k_{\mathrm{pp}}}{\alpha} \sum_{r_{i j}<2 R} \vec{f}_{i j}^{\mathrm{pp}}-\frac{k_{\mathrm{pg}}}{\alpha} \vec{f}_{i}^{\mathrm{pg}}, \\
i=1, \ldots, N .
\end{gathered}
$$

The first order differential equation system is solved numerically to obtain the trajectories of the particles [16]. Since no stochastic forces are introduced in the equation of motion, Eq. (11), the trajectories of the particles are completely deterministic. In our simulations we took $R, \mu$, and $\alpha$ to be unity, and we chose $k$ such that no significant overlap occurs. This implies that the physical quantities for the present experimental situation are measured in the following units: distance $R=27 \mu \mathrm{m}$, magnetic field $B_{0}=\mu / R^{3}=650 \mathrm{G}$, force $F_{0}=\mu^{2} / R^{4}=3.1$ dyn, velocity $v_{0}=F_{0} / \alpha=12 \mathrm{~cm} / \mathrm{s}$ (where $\alpha=0.26 \mathrm{~g} / \mathrm{s})$, energy $E_{0}=\mu^{2} / R^{3}=8 \times 10^{-3} \mathrm{erg}$, and time $t_{0}=R / v_{0}=2.3 \times 10^{-4} \mathrm{~s}$.

Since the external magnetic field $\vec{B}$ is assumed to be homogeneous, it affects the translational motion only through its influence on the dipole orientation. In the simulation, the rotational degrees of freedom are not treated dynamically, instead, for each spatial configuration a self-consistent relaxation algorithm is applied to find the equilibrium orientation of dipoles, where each dipole points towards the local (dipolar plus external) magnetic field

$$
\vec{B}_{\mathrm{tot}}\left(\vec{r}_{i}\right)=\sum_{i \neq j=1}^{\mathrm{N}} B_{\mathrm{d}}^{j}\left(\vec{r}_{i}\right)+\vec{B}
$$




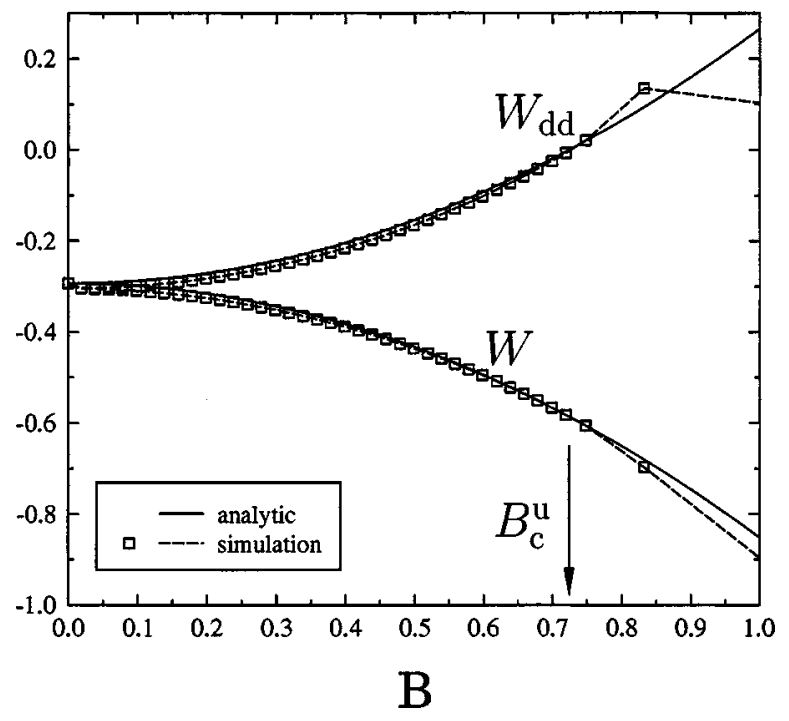

FIG. 7. Comparison of the analytic results with computer simulations for a ring of 15 particles. The value of the upper critical field $B_{\mathrm{c}}^{\mathrm{u}}$ is indicated by an arrow. The analytic and numerical results agree well up to $B_{\mathrm{c}}^{\mathrm{u}}$ as expected.

where $\vec{B}_{\text {tot }}\left(\vec{r}_{i}\right)$ is the total magnetic field at the position of particle $i, B_{\mathrm{d}}^{j}\left(\vec{r}_{i}\right)$ is the dipolar field of particle $j$ at the position of $i$, and $B$ denotes the homogeneous magnetic field imposed externally. After each integration step of the equation of motion, Eq. (11), the dipole moments of the particles are turned to be parallel to the local magnetic field $\vec{B}_{\text {tot }}$. It is iterated until equilibrium is achieved. The method corresponds to the limiting case of infinite rotational mobility, when the dipole orientations are in equilibrium in any moment. This simulation technique has been successfully applied to study the formation of rings of dipoles in the absence of an external magnetic field [12], and their breakup in a magnetic field parallel to the ring's plane [13].

\section{COMPARISON OF EXPERIMENTS, SIMULATIONS, AND ANALYTIC CALCULATIONS}

In the simulations, the particles were placed initially on a circle such that the particle system is in equilibrium in the absence of an external field, see also Fig. 4. Then the external magnetic field $B$ was imposed perpendicular to the plane of the ring. When the field was gradually increased the dipoles rotated in the ring up to reaching the upper critical field $B_{\mathrm{c}}^{\mathrm{u}}$, without any distortion of the ring's structure. For a ring of 15 particles the energy of the dipole-dipole interaction $W_{\mathrm{dd}}$ and the total energy $W$ of the ring was monitored as a function of $B$, which is compared to the analytic solution in Fig. 7. Nice agreement of the analytical and numerical results can be observed until the upper critical field $B_{\mathrm{c}}^{\mathrm{u}}$ is reached, where the particle system becomes unstable and reorganizes itself achieving equilibrium in the simulations. The total interaction energy $W$ of the system monotonically decreases during the entire process as expected.

Since in the simulations the dipoles always take their deepest energy conformation, there is only a slight difference between the final results of gradually increasing the magnetic field up to a certain value or suddenly imposing the same value. Snapshots of the evolution of the particle system in a simulation, when a field value larger than $B_{\mathrm{c}}^{\mathrm{u}}$ was imposed, are compared to the corresponding experimental results in Fig. 3. In the snapshots obtained by the simulations the orientation of the dipole moments of the particles is indicated by arrows. Since the dipole-dipole interaction cannot overweight the effect of the external field, the dipoles suddenly turn over the critical angle $\Theta_{c}$ and the ring practically explodes, see Figs. 3(b) and 3(h). The external magnetic field is homogeneous, hence the further pattern evolution is solely due to the mutual interaction of dipoles, i.e., the particles with almost parallel dipole moments tend to form short chains aligned with the external field, see Figs. 3(c)-3(f) and 3(i) $-3(1)$.

To study the sensitivity of the ring to external perturbations in the metastable regime, a fixed value of $B$ was imposed falling between $B_{\mathrm{c}}^{1}$ and $B_{\mathrm{c}}^{\mathrm{u}}$, and a perturbation of the ring was introduced so that one particle was slightly displaced from its equilibrium position along the field direction. Snapshots of the time evolution of the perturbed ring are compared to the experimental results in Fig. 2. Since no gravity was implemented in the model the value of $B$ was kept fixed during the simulations, while in the experiments the magnetic field had to be continuously increased in order to get full alignment with $B$. A qualitative agreement of the simulations and experiments can be observed in Fig. 2. Simulations revealed that such perturbations provoke always the spiral opening of the ring, which implies that spiral opening is the lowest energy transition from the ring to the chain conformations.

Substituting the parameter values of Sec. IV into the analytic results of Sec. III, the theoretical prediction of the upper critical field $B_{\mathrm{c}}^{\mathrm{u}}$ for the present experimental setup falls between 357-488 $\mathrm{G}$, and the maximum value of the lower critical field $B_{\mathrm{c}}^{1}$ is about $13 \mathrm{G}$. However, in the experiments $B_{\mathrm{c}}^{\mathrm{u}}$ was found about $40-50 \mathrm{G}$, which is an order of magnitude smaller than the theoretical values. It has been argued in Sec. II that, contrary to the simulations, in the experiments the particles can never form a perfect ring. The sticking friction of particles with the bottom plate and with each other prevents reaching initially the deepest energy state so that the initial ring is distorted and the dipole moments have random orientation about the deepest energy conformation. Hence, in the experiment the opening starts spontaneously at the weakest point of the ring. This freezing of the initial ring in a distorted state of relatively high energy is the reason for the large difference between the measured and calculated values of $B_{\mathrm{c}}^{\mathrm{u}}$. To clarify this point, in the experiment we applied shaking of the container of the system during the formation of the initial ring in the direction perpendicular to the bottom plate in the absence of a magnetic field, similarly to Ref. [12]. A slight shaking can prevent the system from freezing in local energy minima and helps it to reach a deeper energy conformation. The value of $B_{\mathrm{c}}^{\mathrm{u}}$ measured on rings obtained with shaking was significantly larger than the other ones, which justifies the above argument. 


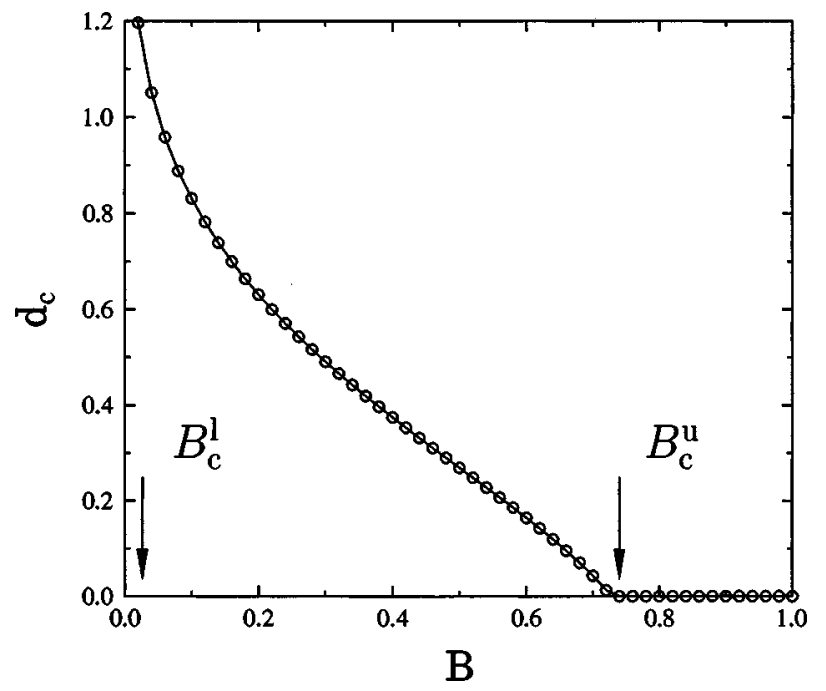

FIG. 8. The critical value of the displacement $d_{c}$ in units of particle diameter $d$ needed to initiate the opening of a ring of 15 particles at a fixed value of $B$.

The sensitivity of the ring to external perturbations depends on the value of the external magnetic field $B$ imposed, i.e., if $B$ is close to $B_{\mathrm{c}}^{\mathrm{u}}$ even a very small amount of perturbation is enough to destroy the ring; however, when $B \approx B_{\mathrm{c}}^{1}$ the ring is rather resistant. To quantify this property of the system, we measured in the simulations the value of the critical displacement $d_{\mathrm{c}}$ of a particle along the field direction needed to provoke an opening at a fixed value of $B$ when $B$ was varied up to $B_{\mathrm{c}}^{\mathrm{u}}$. The results are presented in Fig. 8. It follows from Fig. 8 that for $B \leqslant B_{\mathrm{c}}^{l}$, after the removal of a particle from the ring, the remaining planar chain would close to again form a ring.

To test the effect of the lateral constraint of the hard bottom plate of the vessel on the breakup process, simulations were performed by skipping the last term in the equation of motion, Eq. (11), i.e., by omitting the bottom plate. Experimentally it can be achieved by creating rings of dipoles on a liquid surface into which particles can penetrate during the breakup process. Snapshots of the simulation are presented in Fig. 9. The energetics of the spiral opening with and without lateral constraint is compared in Fig. 10, where $W$ is presented as a function of time during the course of the process for the two cases. Of course, the initial and final energy values are the same in the two cases; however, without lateral constraint the transition occurs along a lower energy path in the phase space, and it takes less time.

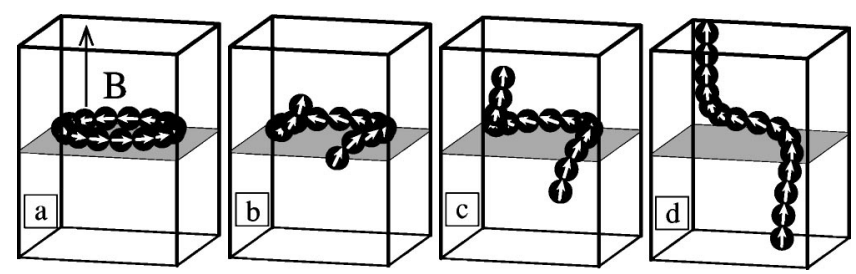

FIG. 9. Spiral opening of a perturbed ring without lateral constraint.

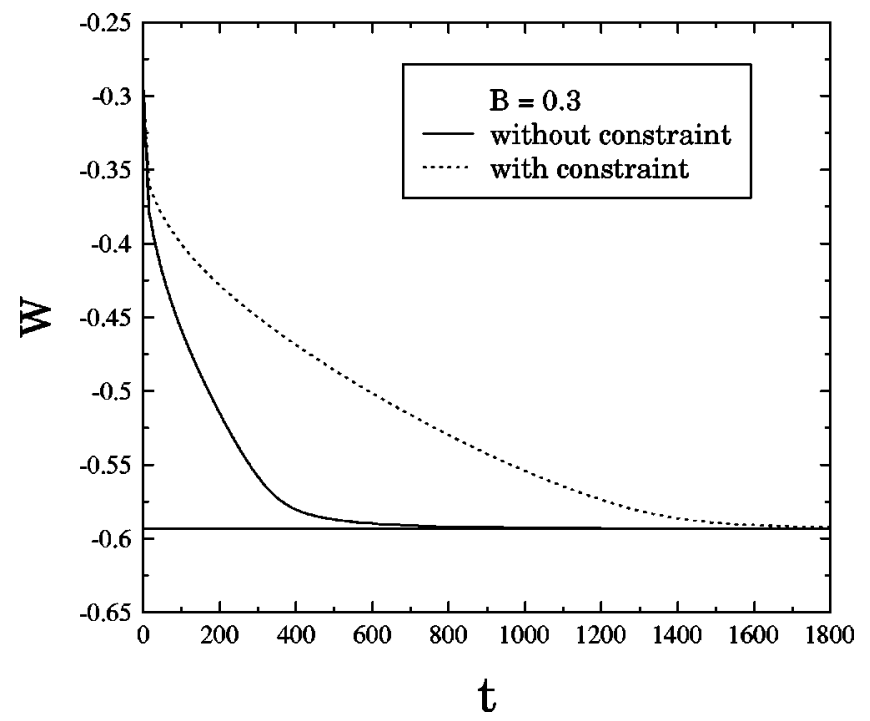

FIG. 10. The energy $W$ as a function of time during the spiral opening process. The full line was obtained without lateral constraint, and the dotted line with lateral constraint due to the hard bottom plate of the vessel. $B$ is measured in the unit of $B_{0}$ and $t$ in the unit of $t_{0}$, respectively.

In the experiments the particles were always magnetized up to the saturation level so that no significant polarization could occur due to the increasing magnetic field. However, the effect of polarization on the breakup process has been considered in the framework of our simulation model, i.e., in the relaxation step of the simulation not only the direction of the dipole moments was changed but also the magnitude according to

$$
\vec{\mu}_{i}=\vec{\mu}_{i}^{0}+\gamma \vec{B}_{\text {tot }}\left(\vec{r}_{i}\right)
$$

where $\vec{\mu}_{\mathrm{i}}^{0}$ denotes the initial dipole moment of particle $i$, and the multiplication factor $\gamma$ depends on the magnetic susceptibility $\chi$ of the material. Simulations have been performed in a wide range of $\chi$ but no significant change of the critical fields $B_{\mathrm{c}}^{1}$, and $B_{\mathrm{c}}^{\mathrm{u}}$ has been observed.

\section{SUMMARY}

If a planar ring of dipoles formed in a suspension is subjected to an external magnetic field the ring rotates until its plane gets perpendicular to the field achieving equilibrium. Hence, to study the breakup of simple structures of a dipolar particle the understanding of the behavior of a dipolar ring in a magnetic field perpendicular to its plane is of high importance. We presented a thorough experimental and theoretical investigation of this problem which was first considered in Ref. [14]. We found experimentally that when the value of the external magnetic field $B$ falls below a lower critical field $B_{\mathrm{c}}^{1}$ the dipoles rotate in the ring without any distortion of the ring structure. However, exceeding an upper critical field $B_{\mathrm{c}}^{\mathrm{u}}$ causes a sudden breakup of the ring into short chains aligned with the field. Between the lower and upper critical fields the system is in a metastable state, characterized by a sensitivity to external perturbations. The spiral opening was found to be 
the lowest energy transition from the ring to the chain conformation. We worked out an analytic approach treating long-range interactions correctly, and we could express the characteristic quantities of the breakup process in terms of characteristics of the initial ring. The time evolution of the breakup process was explored by computer simulations. The analytical and numerical calculations are in a good qualitative agreement with the experimental results. We argued that the polarization of the magnetic particles does not affect significantly the value of the critical fields; furthermore, the quantitative discrepancy of the experimental and theoretical results is due to the effect of static friction.

\section{ACKNOWLEDGMENTS}

This work was supported by the Research Contracts No. RGC/HKUST 6152/00P, DAG No. 99/00.SC32, and NSFC No. 19834020. F. Kun acknowledges financial support from the Alexander von Humboldt Stiftung, the Bólyai János Foundation of the Hungarian Academy of Sciences, and the Research Contract No. FKFP 0118/2001.
[1] P.G. de Gennes and P.A. Pincus, Phys. Kondens. Mater. 11, 189 (1970).

[2] A.T. Skjeltorp, Phys. Rev. Lett. 51, 2306 (1983).

[3] G. Helgesen, A.T. Skjeltorp, P.M. Mors, R. Botet, and R. Jullien, Phys. Rev. Lett. 61, 1736 (1988).

[4] G. Helgesen and A.T. Skjeltorp, J. Appl. Phys. 69, 8277 (1991).

[5] R. Pastor-Satorras and J.M. Rubi, Phys. Rev. E 51, 5994 (1995).

[6] N. Vandewalle and M. Ausloos, Phys. Rev. E 51, 597 (1995).

[7] P.I.C. Teixeira, J.M. Tavares, and M.M. Telo da Gama, J. Phys.: Condens. Matter 12, R411 (2000).

[8] E.M. Furst and A.P. Gast, Phys. Rev. Lett. 82, 4130 (1999).

[9] E.M. Furst and A.P. Gast, Phys. Rev. E 61, 6732 (2000).

[10] J.Y. Huang and P.Y. Lai, Physica A 281, 105 (2000).

[11] J.Y. Huang and P.Y. Lai, Chin. J. Phys. (Taipei) 38, 962 (2000).
[12] W. Wen, F. Kun, K.F. Pál, D.W. Zheng, and K.N. Tu, Phys. Rev. E 59, R4758 (1999).

[13] F. Kun, K.F. Pál, Weijia Wen, and K.N. Tu, Phys. Lett. A 277, 287 (2000).

[14] P. Jund, S.G. Kim, D. Tománek, and J. Hetherington, Phys. Rev. Lett. 74, 3049 (1995).

[15] W.Y. Tam, G. Yi, W. Wen, H. Ma, M.M.Y. Loy, and P. Sheng, Phys. Rev. Lett. 78, 2987 (1997); W. Wen and K. Lu, Phys. Fluids 9, 1826 (1997).

[16] M.P. Allen and D.J. Tildesley, Computer Simulation of Liquids (Clarendon Press, Oxford, 1994).

[17] S. Luding, in Physics of Dry Granular Media, NATO Advanced Study Institute, Series B: Physics, edited by H. J. Herrmann, J-P Hovi, and S. Luding (Kluwer Academic Publishers, Dordrecht, 1998). 